\section{Enfoque comunicativo del Planeamiento Estratégico Situacional en el nivel local: salud y equidad en Venezuela}

\author{
Communicative approach of Situational Strategic \\ Planning at the local level: health and equity \\ in Venezuela
}

\begin{abstract}
1 Instituto de Altos Estudios de Salud Pública "Dr. Arnoldo Gabaldón", Ministerio del Poder Popular para la Salud, Maracay, Venezuela.

2 Escola Nacional de Saúde Pública Sergio Arouca, Fundação Oswaldo Cruz, Rio de Janeiro, Brasil.

Correspondencia E. Artmann Departamento de Administração e Planejamento em Saúde, Escola Nacional de Saúde Pública Sergio Arouca, Fundação Oswaldo Cruz. Rua Leopoldo Bulhões 1480, Rio de Janeiro, $R J$ 21041-210, Brasil. artmann@ensp.fiocruz.br
\end{abstract}

\begin{abstract}
The article discusses the results of operationalizing Situational Strategic Planning adapted to the local level in health, considering the communicative approach and equity in a parish in Venezuela. Two innovative criteria were used: estimated health needs and analysis of the actors' potential for participation. The problems identified were compared to the corresponding article on rights in the Venezuelan Constitution. The study measured inequalities using health indicators associated with the selected problems; equity criteria were incorporated into the action proposals and communicative elements. Priority was assigned to the problem of "low case-resolving capacity in the health services network", and five critical points were selected for the action plan, which finally consisted of 6 operations and 21 actions. The article concludes that the combination of epidemiology and planning expands the situational explanation. Incorporation of the communicative approach and the equity dimension into Situational Strategic Planning allows empowering health management and helps decrease the gaps from inequality.
\end{abstract}

Health Planning; Social Inequity; Social Conditions; Citizen Participation
Henny Luz Heredia-Martínez 1 Elizabeth Artmann 2 Silvia Marta Porto ${ }^{2}$

\section{Introducción}

El Planeamiento Estratégico Situacional (PES) es un enfoque de planificación diseñado para la administración pública ${ }^{1}$. En el contexto internacional, se sitúa dentro de las vertientes tanto estratégica, como en la denominada gestión pública por resultados. En América Latina se inserta en la vertiente crítica al planeamiento tradicional o normativo 2,3 .

Matus 4 define el PES como un método de planeamiento de alta potencia, para procesar problemas mal estructurados y complejos, que considera múltiples dimensiones (política, económica, social, entre otras), así como los diversos actores involucrados en una situación de poder compartido. Según esta concepción, los problemas son generados socialmente, considerándose la realidad una construcción social histórica.

Algunos autores 3,5,6,7,8 han realizado una relectura crítica del PES a partir de la teoría del $a c$ to comunicativo de Habermas 9 , con la finalidad de rescatar y valorar los aspectos comunicativos presentes en la obra de Matus, como el concepto de situación, donde se valoran las diferentes versiones sobre la realidad y la incorporación de las diferencias en un diálogo dirigido al consenso para la construcción de un plan. El conflicto no desaparece, sino que se busca tematizarlo 7 .

En esta experiencia aplicamos el PES dentro de una perspectiva comunicativa, que reconoce el lenguaje como un medio para llegar al enten- 
dimiento, como base de la gestión participativa, como vehículo e instrumento de cooperación y compromiso social 3,9.

Se incorporaron estrategias para rescatar la equidad en los diferentes momentos del PES, mediante la identificación y análisis de los problemas, dando prioridad a las poblaciones menos favorecidas. En este trabajo éste es un punto central, además, las propuestas fueron discutidas teniendo como referencia también los objetivos planteados en las leyes venezolanas [Constitución de la República Bolivariana de Venezuela (1999), Ley Orgánica del Sistema de Seguridad Social (2002), Proyecto de Ley de Salud y Sistema Público Nacional de Salud (2006), Plan Nacional de Desarrollo Económico y Social (2001) y Plan Estratégico Social (2002)]. Entendida la equidad como la igualdad de oportunidades en el uso de servicios de salud, incorporando la igualdad en el acceso, en el tratamiento y en la calidad del mismo 10,11,12.

Consideramos la categoría equidad en todos los momentos del método. Utilizamos los criterios de equidad en la selección de la parroquia (unidad territorial menor que el municipio), en base a la propuesta de Porto 13,14,15. Consideramos dos propuestas, que incorporan tanto elementos comunicativos, como criterios de equidad en el procesamiento de los problemas: (a) la propuesta simplificada para la aplicación del PES en el nivel local en salud, que considera la valoración de los aspectos comunicativos en el planeamiento, a partir de las aportaciones de Habermas 1,6,16; (b) el instrumento de identificación y representación de las necesidades sociales [Instrumento de Identificación y Representación de Necesidades Sociales], desarrollado y aplicado por el Ministerio del Poder Popular para la Salud (MPPS) de Venezuela 17. Finalmente, se consideraron aportes de varios autores de las areas de epidemiología y gestión en salud 19,19,20,21,22, destacándose: el análisis de la situación de salud como herramienta fundamental para el estudio de las interacciones entre las condiciones de vida y los procesos de salud.

Los objetivos del estudio fueron utilizar una adaptación del enfoque del PES para el nivel local en salud, considerando el enfoque comunicativo, e incorporar directamente al método la dimensión de la equidad, apoyada en la aplicación concreta del enfoque en una de las parroquias del Estado de Aragua, Venezuela.

La importancia de esta experiencia se inserta tanto en la dimensión teórico-metodológica como en la práctica, pues con el surgimiento de los consejos comunales y la exigencia de su incorporación activa en el proceso de planeamiento en salud, se hace necesario el uso de herramientas que garanticen la participación de los diferentes actores. Este enfoque puede ser aplicado a otras experiencias en países que pretendan mejorar la equidad en salud.

\section{Materiales y métodos}

La investigación tuvo dos fases: la primera, apoyada en la revisión bibliográfica, consideró la adaptación del PES al nivel local 1,6,16; el Instrumento de Identificación y Representación de Necesidades Sociales, para identificar y representar las necesidades sociales, analizar las situaciones problemáticas de las poblaciones, y proponer medidas de intervención para reducir las inequidades en salud 17; la incorporación de elementos comunicativos, a partir de Habermas 3,5,6,7,8,9,23,24; y la propuesta de Porto et al. $13,14,15$, que incorpora el análisis multivariado de un conjunto de variables epidemiológicas y socioeconómicas del estado estudiado para crear un indicador de necesidades relativas de salud. La segunda fue la aplicación del PES con las modificaciones incorporadas en la parroquia seleccionada, en el período de enero a junio de 2007, a través de talleres con actores claves seleccionados y la inclusión directa de uno de los investigadores.

\section{Criterios de selección de la unidad de análisis}

El Estado de Aragua fue seleccionado por pertenecer a la región central del país, que concentra más del 33\% de la población nacional; es el sexto estado con mayor población de Venezuela (1.689.056 habitantes) y uno de los que presenta mayor desarrollo industrial, conformado por 18 municipios autónomos y 50 parroquias (Instituto Nacional de Estadística. Proyección del censo 2001. http://www.ine.gov.ve, accedido en Nov/2008). A pesar de no ser uno de los estados más pobres (Instituto Nacional de Estadística. Reporte social por estado-2004. http://www.ine. gov.ve, accedido el Ago/2006; Instituto Nacional de Estadística. Mapa de la pobreza. http://www. ine.gov.ve, accedido el Feb/2007), fue seleccionado por las fortalezas existentes en la red de servicios de salud y en el sistema de información, como forma de garantizar la viabilidad del trabajo. De las 50 parroquias, se escogió la parroquia de Capital Santos Michelena, considerando dos criterios de inclusión: (1) que la parroquia tuviese mayores necesidades relativas de salud, según el indicador compuesto de variables epidemiológicas y socioeconómicas y, (2) que los actores-clave tuviesen mayor motivación y potencial partici- 
pativo para garantizar la viabilidad del plan que debía ser diseñado.

\section{- Análisis para la estimativa de las necesidades relativas de salud}

Se utilizó la propuesta de Porto et al. 13,14,15 para contextos de grandes desigualdades. Se construyó un indicador para estimar las necesidades, a partir de un subconjunto de tres variables epidemiológicas y socioeconómicas, de la base de datos con 50 unidades geográficas (parroquias): Tasa de Mortalidad Infantil (TMI) del quinquenio 2001-2005 (por mil nacidos vivos); porcentaje de analfabetos ( $\geq 15$ años); y porcentaje de domicilios sin abastecimiento de agua por acueducto o tuberías que, según varios autores $14,15,26,26,27$ son los que mejor expresan las necesidades de salud. El indicador fue obtenido a través de un procedimiento de análisis estadístico multivariado de los componentes principales. Con la finalidad de evitar las fluctuaciones en la TMI, debido al problema de pequeños números, y para garantizar la calidad del cálculo del índice de necesidades relativas de salud, se utilizaron los siguientes criterios: trabajar con un quinquenio (2001-2005); retirar del análisis aquellas parroquias que tuvieron una media inferior a 100 nacidos vivos, para el quinquenio 2001-2005. De las 50 parroquias, 38 se incorporaron al análisis. No obstante, el resultado de este procedimiento no indica que el PES no pueda ser utilizado en las parroquias que fueron excluidas. Cabe destacar que el propósito de esta fase del análisis fue obtener un índice de necesidades relativas de salud, que mejor expresase la realidad de las parroquias, con la finalidad de alcanzar mayor equidad en la selección de la parroquia, a partir de una priorización técnica.

Así, se obtuvieron dos nuevos factores. En el factor 1, cargas grandes y positivas se encontraron referentes a la tasa de mortalidad infantil y el porcentaje de domicilios sin agua corriente. En el factor 2 el porcentaje de analfabetos presenta carga positiva y próxima a uno. Por ser el factor 1 el que mejor expresa las desigualdades relativas entre las unidades analizadas, se trabajó con los valores correspondientes a ese factor. Se destaca que la tasa de mortalidad del quinquenio representa un 50,6\% de la variación total original (Tabla 1).

En la Tabla 2 se presentan las parroquias con el valor del índice de necesidades relativas de salud. Las cinco parroquias con más necesidades relativas fueron: Capital Santos Michelena, San Martín de Porres, Zuata, Augusto Mijares y Capital Libertador.

\section{- Criterios de selección y análisis del potencial participativo de los actores- clave}

En las cinco parroquias con más necesidades relativas se analizó el potencial participativo de los actores-clave frente al desarrollo del plan. Fueron considerados actores-clave: (1) aquellos que dominan recursos críticos (representante de la Junta Parroquial o del Gobierno Municipal; representante de la Coordinación Municipal de la Misión Barrio Adentro -esta misión surge en Venezuela como una experiencia piloto, y se convierte en el eje articulador de las políticas sociales y de la construcción del Sistema Público Nacional de Salud en Venezuela -; miembros del equipo municipal de salud; representante del sector educativo); (2) los afectados por los problemas (representantes de los Consejos Comunales; representantes de los Comités de Salud). En la investigación participó un total de 70 sujetos, en diferentes momentos.

Se utilizaron los siguientes instrumentos: matriz de motivación para actores que dominan recursos críticos propuesta por Matus 4, cuestionario semi-estructurado basado en criterios

Tabla 1

Análisis de componentes principales

\begin{tabular}{|c|c|c|}
\hline \multirow[t]{2}{*}{ Indicadores utilizados } & \multicolumn{2}{|c|}{ Factores } \\
\hline & 1 & 2 \\
\hline Tasa de mortalidad (quinquenio 2001-2005) (por 1.000 nacidos vivos) & 0,888 & $-0,144$ \\
\hline Porcentaje de analfabetos ( $\geq 15$ años) & 0,046 & 0,974 \\
\hline Porcentaje de domicilios sin abastecimiento de agua por acueducto o tuberías & 0,814 & 0,313 \\
\hline
\end{tabular}

Fuente: a partir de datos tomados del censo 2001 (Instituto Nacional de Estadística. Proyección del censo 2001. http://www. ine.gov.ve, accedido el Nov/2008) y del Sistema de Información del Modelo de Atención Integral (SISMAl, 2001-2005). 
Tabla 2

Índice de necesidades para las parroquias del Estado de Aragua, Venezuela, año 2007.

\begin{tabular}{|c|c|c|c|}
\hline $\mathbf{N}^{\circ}$ & Parroquia & Municipio & Valores (entre 1 y 2 ) * \\
\hline 1 & Joacuín Crespo & Girardot & 1,00 \\
\hline 2 & Capital Camatagua & Camatagua & 1,05 \\
\hline 3 & Caña de Azúcar & Mario Briceño Iragorry & 1,16 \\
\hline 4 & Francisco de Miranda & Francisco Linares Alcántara & 1,19 \\
\hline 5 & Bella Vista & Sucre & 1,22 \\
\hline 6 & Capital San Casimiro & San Casimiro & 1,22 \\
\hline 7 & Pedro José Ovalles & Girardot & 1,24 \\
\hline 8 & Capital Francisco Linares Alcántara - Santa Rita & Francisco Linares Alcántara & 1,25 \\
\hline 9 & Las Delicias & Girardot & 1,27 \\
\hline 10 & Arévalo Aponte & Mariño & 1,29 \\
\hline 11 & Los Tacariguas & Girardot & 1,32 \\
\hline 12 & Capital José Angel Lamas & Lamas & 1,33 \\
\hline 13 & Alfredo Pacheco Miranda & Mariño & 1,33 \\
\hline 14 & Valles de Tucutunemo & Zamora & 1,33 \\
\hline 15 & Las Guacamayas & Ribas & 1,35 \\
\hline 16 & José Casanova Godoy & Girardot & 1,37 \\
\hline 17 & Capital San Sebastián & San Sebastian & 1,39 \\
\hline 18 & Samán de Güere & Mariño & 1,39 \\
\hline 19 & Castor Nieves Ríos & Ribas & 1,40 \\
\hline 20 & Andrés Eloy Blanco & Girardot & 1,41 \\
\hline 21 & San Francisco de Asís & Zamora & 1,41 \\
\hline 22 & Magdaleno & Zamora & 1,44 \\
\hline 23 & José Rafael Revenga & Revenga & 1,45 \\
\hline 24 & Madre María de San José & Girardot & 1,46 \\
\hline 25 & Ocumare de La Costa de Oro & Costa De Oro & 1,46 \\
\hline 26 & Capital José Félix Ribas & Ribas & 1,49 \\
\hline 27 & Monseñor Feliciano González & Francisco Linares Alcántara & 1,49 \\
\hline 28 & Capital Sucre & Sucre & 1,50 \\
\hline 29 & Capital Santiago Mariño & Mariño & 1,50 \\
\hline 30 & Capital Mario Briceño Iragorry & Mario Briceño Iragorry & 1,52 \\
\hline 31 & Capital Tovar & Tovar & 1,54 \\
\hline 32 & Capital Bolívar - San Mateo & Bolívar & 1,57 \\
\hline 33 & Capital Zamora & Zamora & 1,63 \\
\hline 34 & Capital Santos Michelena & Santos Michelena & 1,65 \\
\hline 35 & San Martín de Porres & Libertador & 1,80 \\
\hline 36 & Zuata & Ribas & 1,82 \\
\hline 37 & Augusto Mijares & Zamora & 1,91 \\
\hline 38 & Capital Libertador & Libertador & 2,00 \\
\hline
\end{tabular}

Fuente: censo 2001 (Instituto Nacional de Estadística. Proyección del censo 2001. http://www.ine.gov.ve, accedido el Nov/2008) y del Sistema de Información del Modelo de Atención Integral (SISMAI, 2001-2005).

* Los cálculos fueron realizados a partir de la tasa de mortalidad quinquenio 2001-2005 (por 1.000 nacidos vivos), el porcentaje de analfabetos ( $\geq 15$ años) y el porcentaje de domicilios sin abastecimiento de agua por acueducto o tuberías. El valor varía entre 1 y 2 ( 1 con menos necesidades relativas y los más próximos a 2 con más necesidades). 
propuestos por Carvalho 28 y otros, con la finalidad de ampliar la información de la matriz de motivación de los actores y captar su capacidad de acción, así como, conocer su nivel de organización. El cuestionario permitió abordar algunas limitaciones de la matriz de motivación de los actores-clave 3,29 comprobando la información recogida. Los criterios considerados fueron: la capacidad de convocatoria para involucrar a otros actores, así como contar con información automatizada y disgregada hasta el nivel de localidades.

Para el análisis de ambos instrumentos se calcularon frecuencias y porcentajes para cada uno de los rubros. Así, el 80\% de los actores de las parroquias pre-seleccionadas en el análisis del indicador compuesto de necesidades relativas, designaron un valor entre medio y alto al desarrollo del proyecto. Después del análisis efectuado, la parroquia seleccionada fue Capital Santos Michelena teniendo en cuenta que: los actoresclave tuvieron la mayor motivación y potencial participativo para desarrollar el proyecto, incluyendo la principal autoridad del municipio y de esa parroquia; contaba con un alto porcentaje de comités de salud y consejos comunales; el director municipal de salud tenía un alto poder para convocar a los diferentes actores-clave y manifestó estar a favor del desarrollo del proyecto; se contaba con información socio-epidemiológica actualizada hasta 2007 y disgregada hasta el nivel de localidad.

\section{Propuesta del PES para el nivel local en salud en Venezuela}

Fue considerada la propuesta que adapta el PES al nivel local en salud en su enfoque comunicativo e incorporando la dimensión de la equidad. Se trabajaron: el momento explicativo; el normativo y el estratégico. El momento táctico-operacional no fue analizado en este trabajo.

\section{- Momento explicativo}

En los talleres con los actores-clave se priorizó la participación de personas conocedoras de la realidad, pertenecientes a diferentes sectores (habitantes de la parroquia, consejos comunales, consejos de salud, equipo de educación, equipo de salud, entre otros). La argumentación fue utilizada como estrategia para garantizar la participación de diversos actores, con el objetivo de alcanzar la búsqueda del entendimiento en el sentido habermasiano ${ }^{9} \mathrm{y}$ teniendo siempre como eje central contribuir, de la forma más equitativa posible, a la modificación de la situación problemática. Fue utilizado un diario de campo en todas las actividades desarrolladas para registrar información y declaraciones de los actores sociales para el proceso de análisis. Comprendió los siguientes pasos:

\section{(a) Selección del problema}

Combinándose la técnica "tormenta de ideas" con argumentación se identificaron los problemas de salud y cada uno de ellos se relacionó con los artículos de la Constitución de la República Bolivariana de Venezuela que no estaban siendo cumplidos integralmente 17 . Se proporcionó una lista de los principales artículos de la Constitución, especificándose los derechos relacionados con cada uno y los temas de cada artículo. Después los problemas fueron jerarquizados a partir de la aplicación de un protocolo simple de selección con la participación de los actores con control de los recursos críticos.

\section{(b) Descripción del problema priorizado}

A través de indicadores cuantitativos o cualitativos de determinantes de la salud: natalidad, morbilidad, mortalidad, entre otros ${ }^{4}$. Se utilizaron los registros de las bases de datos: (a) del Instituto Nacional de Estadística de Venezuela (INE); (b) del Sistema de Información de Salud de la Corporación de Salud del Estado de Aragua (CORPOSALUD); (c) del Sistema de Vigilancia Alimentaria y Nutricional.

\section{(c) La explicación situacional}

A través de talleres se construyó el flujograma situacional, basado en la Teoría de la Producción Social 30,31,32, considerando los descriptores (VDP), las causas del problema en una cadena multicausal y las consecuencias. Se estratificaron las localidades, se midieron las inequidades sociales (según el instrumento desarrollado por el MPPS) y se actualizó el Análisis de la Situación de Salud 19.

En la estratificación de las localidades se consideró un total de 27 variables para la construcción de un indicador de condiciones de vida de la población, a partir del cual fueron clasificadas en cuatro estratos o territorios sociales según condiciones de vida desde 1 (mejor) hasta 4 (peor), en las categorías de condiciones de vida: buenas, regulares, malas y muy malas. Los pesos ponderados de las variables se sumaron a cada unidad de análisis (localidad), y se conformaron los intervalos para estratificar las localidades, utilizando el procedimiento de Conglomerados K-Medias. Fue utilizada la guía de observación del Instituto de Altos Estudios de Salud Pública "Dr. Arnoldo 
Gabaldón” (IAES), y del MPPS de 2002, en las 42 localidades de la parroquia.

\section{(d) Selección y descripción de los nudos críticos}

A través de talleres con los actores, todas las causas se evaluaron según los criterios: tener alto impacto sobre el VDP (descriptores); ser un centro práctico de acción; oportunidad política de acción 4,30,32, fueron seleccionadas aquellas que respondieron positivamente (nudos críticos). Se identificaron los actores que controlan las variables de cada nudo crítico, para verificar el grado de gobernabilidad del que depende el plan de acción.

\section{- Momento normativo}

Fue diseñado el plan de acción con los actoresclave, considerando elementos de la propuesta de Artmann 6. La situación-objetivo fue conformada como vector de descripción de resultados o metas terminales e intermediarias. Se procedió a la construcción de tres escenarios posibles (optimista, intermedio y pesimista), pero se escogió el escenario intermedio para delinear el programa direccional (operaciones, productos, resultados, responsables, recursos y tiempo de realización). Para garantizar que el plan incluyese criterios de equidad, fueron priorizadas operaciones y acciones que beneficiaran a las poblaciones más vulnerables, con el fin de contribuir a la disminución de las inequidades. Aquí se utilizó toda la información producida en el momento explicativo.

\section{- Momento estratégico}

Se analizó la viabilidad del plan en sus diferentes dimensiones: política, económica, cognitiva y organizativa, en base a: (a) motivación de los actores frente a las operaciones del plan, con la finalidad de registrar el interés y el valor que atribuyen al plan; (b) capacidad de acción para determinar el grado de control de los recursos críticos necesarios en la implementación de las operaciones y acciones por parte del grupo a favor y en contra del plan. Se construyeron estrategias para las acciones conflictivas, dando prioridad a la negociación cooperativa como medio para alcanzar los objetivos del plan.

Esta investigación fue aprobada por el Comité de Ética en Investigación de la Escuela Nacional de Salud Pública Sergio Arouca, Fundación Oswaldo Cruz, en 2007.

\section{Resultados y discusión}

En el primer taller de trabajo se identificaron los cinco problemas de salud más importantes (Tabla 3), a partir de un debate donde los actores se mostraron con dominio del asunto y rebatían con argumentos o aceptaban la explicación de los otros actores, incluso cuando eran algunas de las autoridades de salud quienes manifestaban una opinión contraria. Reflexionar sobre la relación de cada uno de los problemas con los artículos de la Constitución de la República Bolivariana de Venezuela, llamó la atención de varios actores en el sentido de que ultrapasaban situaciones particulares y afectaban los derechos establecidos en la carta magna.

La forma en la que cada actor argumentaba la importancia sobre los problemas que vive, mostró diferentes puntos de vista: para los representantes de los consejos comunales, la baja capacidad resolutiva de los servicios de salud era un problema fundamental; para los representantes del equipo de salud existían otras prioridades, tales como inseguridad, falta de agua, problemas de recolección de basura. Sin embargo, uno de los habitantes dio una explicación que, por la fuerza del argumento, convenció a los otros sobre la prioridad del mismo. Se reconoció que en la parroquia existían otros problemas, no obstante, estos se desdoblaban y generalmente sus consecuencias eran atendidas por el sector de salud, por tanto, tener garantizado ese servicio era una prioridad.

Se dio prioridad a la "baja capacidad resolutiva de los servicios de salud" argumentándose que: afectaba a toda la población de la parroquia, y más aún a las poblaciones con menos recursos; con su solución se daría respuesta a dos de los cinco problemas principales: "atención médica las 24 horas insuficiente para cubrir las demandas de la parroquia" y "déficit en la distribución de medicamentos esenciales", que pueden ser considerados problemas derivados de aquel. Parte del problema está bajo control de la Dirección Municipal de Salud de la parroquia, por tanto, dentro de su espacio de gobernabilidad 4 . Los otros problemas se quedaron en el archivo de problemas de los actores.

La participación del investigador como mediador en la discusión también fue un elemento importante que permitió un puente entre los conocimientos de la comunidad y los conocimientos del equipo de salud.

Se construyó el flujograma situacional en el segundo taller, que fue revisado en el tercer taller con reajustes, según observaciones de un experto en planeamiento. Se discutió cada detalle para llegar a un acuerdo. 
Tabla 3

Priorización de los problemas y su relacción con los artículos constitucionales no cumplidos integralmente. Parroquia Capital, Municipio Santos Michelena, Estado de Aragua, Venezuela, año 2007.

\begin{tabular}{|c|c|c|c|c|c|c|c|c|}
\hline $\mathbf{N}^{\circ}$ & Problemas & $\begin{array}{l}\text { Artículos de la } \\
\text { Constitución }\end{array}$ & $\begin{array}{c}\text { Valor } \\
(\mathrm{A}, \mathrm{M}, \mathrm{B}, \mathrm{O})\end{array}$ & $\begin{array}{c}\text { Costo } \\
\text { económico } \\
(A, M, B, 0)\end{array}$ & $\begin{array}{c}\text { Costo de } \\
\text { postergación } \\
(A, M, B, 0)\end{array}$ & $\begin{array}{c}\text { Eficacia en la } \\
\text { intervención } \\
(A, M, B, 0)\end{array}$ & $\begin{array}{c}\text { Gobernabilidad } \\
\text { (A, M, B, 0) }\end{array}$ & Selección S/N \\
\hline 1 & $\begin{array}{c}\text { Déficit en la } \\
\text { distribución de } \\
\text { medicamentos }\end{array}$ & $\begin{array}{c}80,82,83,84 \\
85,86,122\end{array}$ & M & A & A & B & B & $\mathrm{S}$ \\
\hline 2 & $\begin{array}{c}\text { Atención } \\
\text { médica } \\
24 \text { horas } \\
\text { insuficiente } \\
\text { para cubrir las } \\
\text { demandas de } \\
\text { la parroquia }\end{array}$ & $76,83,84,86$ & A & M & A & M & M & $\mathrm{S}$ \\
\hline 3 * & $\begin{array}{c}\text { Baja } \\
\text { capacidad } \\
\text { resolutiva } \\
\text { de la red de } \\
\text { servicios de } \\
\text { salud }\end{array}$ & $76,83,84,86$ & A & A & A & A & M & $\mathrm{S}$ \\
\hline 4 & $\begin{array}{l}\text { Inadecuada } \\
\text { disposición } \\
\text { de residuos } \\
\text { sólidos }\end{array}$ & $107,127,129$ & A & B & A & M & $\mathrm{M}$ & $\mathrm{S}$ \\
\hline 5 & $\begin{array}{c}\text { Racionamiento } \\
\text { y mala calidad } \\
\text { del agua } \\
\text { que se le } \\
\text { suministra al } \\
\text { municipio }\end{array}$ & $107,127,129$ & A & M & A & M & $M$ & $\mathrm{~S}$ \\
\hline
\end{tabular}

Fuente: primer taller con los diferentes actores sociales de la Parroquia Capital, Municipio Santos Michelena, Estado de Aragua, Venezuela, abril 2007.

Leyenda: A: alto; M: medio; B: bajo; 0: cero; S: sí; N: no.

* Problema seleccionado

Las causas fueron clasificadas según la gobernabilidad de los actores implicados, encontrándose algunas comunes a varios problemas del sector salud, fundamentales en la explicación intersectorial, pese a que estaban fuera del espacio del problema 4,32 .

El flujograma situacional exigió discutir y explicar cuestiones de la realidad bajo diferentes puntos de vista, para lo que se hace fundamental el enfoque comunicativo. Una observación importante de uno de los actores del nivel de estados que participó en el diseño del plan, fue que, aunque fueron consideradas en un contexto local, las causas del problema de la baja capacidad resolutiva de los servicios de salud en la parroquia Capital Santos Michelena son similares a lo que sucede en el Estado de Aragua y en el país.
Se seleccionaron los nudos críticos, objetivos de la intervención, utilizándose la matriz de valoración de Matus 4 . Es de resaltar, que en el análisis realizado la resolución de las causas estructurales dependía de otros niveles de gobernabilidad superior al de los actores-clave. Así, los actores reflexionaron sobre la importancia del concepto de espacio de gobernabilidad, y la necesidad de construcción de un plan de demandas como Matus defiende para los problemas cuyas causas se encuentran fuera de la gobernabilidad del actor local o principal 4,32 .

Se seleccionaron los siguientes nudos-críticos: (1) insuficiente organización y participación comunitaria en acciones de promoción de salud; (2) desintegración de las acciones en los diferentes niveles de atención de la red de salud; (3) frag- 
mentación, desarticulación de la red de sistemas y servicios de salud; (4) insuficiente número de recursos humanos cualificados en algunos niveles de atención; y (5) baja capacidad tecnológica.

\section{La otra cara de la explicación situacional:} una perspectiva desde la equidad

En paralelo a los talleres, se realizaron visitas para la aplicación de la guía de observación en las 42 localidades de la parroquia, lo que fue fundamental para el proceso de planeamiento, pues permitió conocer cada localidad, observar las principales condiciones de vida que las caracterizan, intercambiar información con otros actores-clave que no participaron en los talleres, recabar información desde la fuente primaria y realizar el mapeo.

Se analizaron los datos recolectados en el Sistema de Información en Salud, de la Dirección Municipal de Salud Santos Michelena (DMSSM) y los generados por la Coordinación Municipal de la Misión Barrio Adentro (CMMBA), con la finalidad de ampliar la explicación situacional, buscando identificar las brechas por inequidad que se reproducen en la parroquia.

El equipo de salud en un primer momento no reconoció el problema seleccionado como el más importante. No obstante, al iniciarse el análisis de los datos con una perspectiva desde la equidad, estos reconocieron que la baja capacidad resolutiva de los servicios de salud constituía un problema en la parroquia.

De las 42 localidades, 55\% fueron clasificadas en condiciones de vida malas y muy malas, y sólo un $9 \%$ en condiciones de vida buenas (Tabla 4). Las localidades con condiciones de vida muy malas carecen de servicios básicos, se encuentran en la periferia, son producto de invasiones y crecen sin ningún tipo de planificación. La mayor parte de las localidades con regulares condiciones de vida también están apartadas del centro de las Tejerías, al contrario que las localidades con buenas condiciones de vida, lo que facilita el acceso a los servicios públicos en general.

Los establecimientos de salud en la parroquia se encuentran situados más cerca de las localidades con mejores condiciones de vida, incluyendo los consultorios populares. Las nueve localidades más apartadas (21\%) no cuentan con servicios de salud cerca, sus condiciones de vida varían entre muy malas y malas, son de difícil acceso, o el transporte público no funciona con regularidad.

Todos los Núcleos de Atención Primaria (NAP) y los Ambulatorios Rurales Tipo II en el momento de la investigación estaban sin personal médico. Los habitantes reconocen que la situación ha mejorado, el ambulatorio de mayor complejidad de la parroquia en los últimos cinco años amplió consultas por especialidades, además de habilitar la emergencia 24 horas, se abrieron el Centro de Diagnóstico Integral (CDI) y la Sala de Rehabilitación Integral (SRI), beneficiando a la población más necesitada.

No obstante, el $80 \%$ de los establecimientos dependientes de la Dirección Municipal de Salud en mayo de 2007 estaban sin médicos generales, a pesar de tener disponibles los recursos para la contratación. En tal sentido, para la población la capacidad resolutiva de la red de servicios de salud continúa siendo baja, debido a que de los 18 establecimientos que existen dentro de la parroquia, dos tienen atención 24 horas, siendo uno de ellos (el CDI) más dirigido a los estudios de diagnóstico fundamentales, con un $50 \%$ de los servicios funcionando en el período de estudio.

Del análisis se deduce que parte del problema podría ser resuelto con una redistribución de los recursos humanos en la red de servicios de salud en la parroquia, ampliación de los horarios de atención, habilitación de otros centros con atención las 24 horas, entre otras acciones. Contratar a los médicos de los NAP con mejores salarios es una operación que debe ser desarrollada en el plan de acción.

En la evaluación de la capacidad relativa a recursos humanos en los servicios de salud se observa que apenas un $10 \%$ (4) de las localidades fueron consideradas con buena capacidad en contra del $90 \%$ con baja capacidad. Los actores-clave manifestaron en el primer taller el problema de la falta de médicos para atender a las emergencias después de las 12:00. Hay localidades donde los habitantes quedan aislados, sin comunicación cuando llueve, y sólo cuentan con atención en horario limitado. Observamos que los datos analizados son el reflejo de las opiniones de los habitantes o viceversa.

A través de la guía se consultó a los informantes claves sobre los problemas prioritarios de la localidad para compararlos con los discutidos en el primer taller y así ampliar el archivo de problemas de la parroquia. La falta de agua en las diferentes opiniones de los informantes claves fue resaltada, puesto que un $81 \%$ de las localidades presentan ese problema, en su mayoría, de los estratos 2, 3 y 4. En cuanto a la participación comunitaria, un $83 \%$ de los informantes claves la evaluaron como regular o mala; un $12 \%$ como buena y sólo un $5 \%$ como excelente.

El análisis evidenció que la baja capacidad resolutiva de los servicios de salud en la parroquia es un problema que afecta sobre todo a las poblaciones menos favorecidas. En la redistribución de los recursos humanos se hace necesario 
Conformación de los territorios sociales según condiciones de vida, Parroquia Capital, Municipio Santos Michelena, Estado de Aragua, Venezuela, año 2007.

\begin{tabular}{|c|c|c|c|}
\hline $\begin{array}{l}\text { Territorios sociales } \\
\text { (puntuación final por } \\
\text { estratos) }\end{array}$ & $\begin{array}{l}\text { Condiciones } \\
\text { de vida }\end{array}$ & N (\%) & Localidades \\
\hline I (40-55) & $\begin{array}{l}\text { Condiciones de } \\
\text { vida buenas }\end{array}$ & $4(9)$ & $\begin{array}{c}\text { Urbanización Mendoza, La Cañada Ruiz Pineda, La Cañada el } \\
\text { Mamón y Centro de Las Tejerías }\end{array}$ \\
\hline II (60-90) & $\begin{array}{l}\text { Condiciones de } \\
\text { vida regulares }\end{array}$ & $\begin{array}{c}15 \\
(36)\end{array}$ & $\begin{array}{c}\text { La Estación, Los Alpes, Los Jabillos, Castor Nieves Ríos, } \\
\text { Guaicaipuro, Jabillal, Tierra Amarilla, Los Cachos, Los Límites, } \\
\text { Curiepe, Las Terrazas, Tinapuey II, El Béisbol, La Cañada las } \\
\text { Veras y Valle Alto }\end{array}$ \\
\hline III (95-120) & $\begin{array}{l}\text { Condiciones de } \\
\text { vida malas }\end{array}$ & $\begin{array}{c}18 \\
(43)\end{array}$ & $\begin{array}{c}\text { Tinapuey I, San Luis, La Lomitas, La Pradera, La Línea Primer } \\
\text { Túnel, La Línea Segundo Túnel, La Línea Cuarto Túnel, Antonio } \\
\text { José de Sucre, Santa María, Pueblo Nuevo, Araiza, Guayas, } \\
\text { Libertador, La Línea Tercer Túnel, Boca de Cagua, Tabacal, } \\
\text { Cañaote y Bucaral }\end{array}$ \\
\hline IV (125-140) & $\begin{array}{l}\text { Condiciones de } \\
\text { vida muy malas }\end{array}$ & $5(12)$ & $\begin{array}{l}\text { Llano Grande, Brisas de Aragua (Constituyente), La Arenera, } \\
\text { Bolívar y Morocopo Bajo }\end{array}$ \\
\hline Totales & & $\begin{array}{c}42 \\
(100)\end{array}$ & \\
\hline
\end{tabular}

Fuente: guía de observación aplicada en la Parroquia Capital, Municipio Santos Michelena, Estado Aragua, Venezuela, 2007.

incorporar criterios de equidad, de forma tal que no se reproduzcan las brechas existentes.

\section{El plan de acción}

Se dio prioridad a la atención de las localidades que quedaron clasificadas en los estratos con peores condiciones de vida, considerando la dimensión de la equidad.

Se realizaron talleres con parte del equipo gerencial de CORPOSALUD Aragua, que, además de estar vinculados directamente con la problemática priorizada, poseían conocimientos técnicos para aportar soluciones y serían los responsables del desarrollo del plan en la parroquia. Algunos actores que no participaron directamente fueron

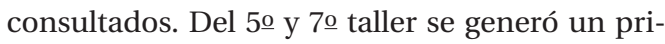
mer documento con las líneas generales del plan y los análisis de los diferentes escenarios.

En el 8o taller se presentó una versión preliminar de las líneas generales del plan de acción, donde se diseñó lo que sería el funcionamiento ideal de la red de los servicios de salud.

En el 9o taller y en las discusiones vía Internet con el equipo que participó en la construcción del plan se diseñaron las operaciones, tomando como referencia la propuesta de Rivera ${ }^{3}$. Se utilizó un concepto de escenario más flexible, dada la necesidad manifestada por los actores, de incluir la motivación de los actores en la configuración de los diferentes escenarios.

Los escenarios fueron construidos teniendo por referencia un período de tiempo de un año, ya que los actores destacaron la fuerte inestabilidad y cambios continuos en el sector salud en el país, característica que dificulta pensar en escenarios que abarquen periodos prolongados 3 . Para el equipo este ejercicio conllevó la discusión sobre la incertidumbre del comportamiento de algunas variables y sobre los diferentes puntos de vista de los diversos actores 4,32 . En el escenario intermedio: se mantiene el actual presupuesto en salud del municipio en torno a US\$ 866.125 (95\% destinados a gastos de recursos humanos y $5 \%$ a gastos de funcionamiento) para atender a una población de 44.409 habitantes (2007); se produce un aumento de un $10 \%$ en el salario de los médicos; los indicadores de morbi/mortalidad y de las condiciones de vida de la población se mantienen; cambian las autoridades de gobierno municipal (alcalde), indicando necesidad de negociación con las nuevas autoridades; el director municipal de salud logra negociar el apoyo para cubrir algunas acciones del plan; se mantienen las actuales autoridades estatales (gobernador y presidente de CORPOSALUD Aragua), con apoyo económico y político al plan de acción. Aumenta la inversión para mejorar la 
infraestructura y equipar un $30 \%$ de los establecimientos de la red; se mantiene el apoyo económico del gobierno nacional en el fortalecimiento de la red de servicios de salud. Hay continuidad en el trabajo intersectorial en la parroquia coordinado por la Dirección Municipal de Salud y la Coordinación Municipal de Barrio Adentro; el equipo de esa coordinación no concuerda con algunas operaciones del plan, pero participa en un $60 \%$ de las operaciones del plan de acción. Es necesario diseñar operaciones de negociación para viabilizar acciones, donde la participación de ellos es punto clave; se consigue una participación activa de por lo menos un $60 \%$ de los consejos comunales en varias de las operaciones del plan, así como en la defensa de éste ante las autoridades.

En relación con el escenario pesimista se pensaron algunas estrategias que permitieran contraponerse a las tendencias negativas: involucrar a los consejos comunales en el plan de acción desde el inicio del proceso de planeamiento, para garantizar la defensa del plan ante las nuevas autoridades, así como el empoderamiento de la comunidad en las acciones del plan y captar algunos recursos por la vía de proyectos, a través de los planes de desarrollo de los consejos; involucrar al equipo de salud con el plan, de forma tal que si el director municipal de salud fuera removido de su cargo existiera una mayor garantía de continuidad del plan; argumentar junto a las autoridades sobre las ventajas del plan de acción como instrumento para la disminución de las brechas por inequidad en la parroquia, destacando las operaciones que fortalecen la capacidad local.

Es bastante probable concretar algunas de las acciones del plan. De hecho, incluso durante los momentos explicativo y normativo, ya se estaban ejecutando algunas actividades como: medidas para mejorar la calidad de la información del Sistema de Información en Salud (SIS); se actualizó la sala situacional y se evidenció la necesidad de potenciarla para que su desarrollo esté direccionado por las políticas, prioridades y compromiso de la gestión en salud, articulando las necesidades y demandas priorizadas por la población con los recursos disponibles 3,18; se generó la demanda de capacitación del equipo de salud en el uso de herramientas para el análisis de la información en salud; el proceso de planeamiento sirvió de apoyo para algunos de los consejos comunales que estaban desarrollando planes de acción; se abrió la discusión sobre cómo incluir criterios más equitativos en la ejecución de los programas de salud, y en la construcción de estrategias para el trabajo en red, entre otras.

Esto confirma lo que Matus 4,32 señala sobre el plan, ya que no se ejecuta una vez que ha si- do diseñado, sino desde la selección de los problemas. Así, el proceso de planeamiento en su enfoque estratégico situacional, comunicativo y creativo se revela dinámico.

Se acordó explicitar en cada operación los criterios de equidad a considerar, ya que lo más probable es que los recursos no serían suficientes para atender a todas las localidades.

No obstante, esta medida no inviabiliza la atención a las poblaciones que están en mejores condiciones, porque existen operaciones que una vez ejecutadas beneficiarán a todas las localidades. Se buscó establecer claramente las poblaciones que son prioritarias para la Dirección Municipal de Salud.

El enfoque comunicativo en la creación de viabilidad del plan es de suma importancia, en el sentido de que éste permite desarrollar estrategias de negociación, donde todos los actores involucrados pueden obtener ganancias 5,6,8. Por eso, los actores que participaron no concordaron en llamar oponentes a aquellos que por alguna razón no apoyaron algunas de las operaciones del plan, considerando la necesidad de utilizar estrategias de negociación, más que para convencer al actor, para discutir abiertamente sus consideraciones y aportes. La necesidad de abrir el debate y discutir los argumentos que son conflictivos ha sido apuntada como fundamental para transformar oponentes en colaboradores 3,33. No reducir los actores con opiniones diferentes o contrarias al plan a meros oponentes abre la perspectiva de negociación, antes del enfrentamiento, lo que puede aumentar las condiciones de viabilidad del plan.

Cabe destacar que, en esta parroquia, las autoridades de salud tienen una trayectoria de negociación con diferentes actores, aspecto que fortalece la viabilidad del plan. A pesar de eso, para varios actores la negociación continúa siendo un gran desafío, más todavía al tratarse de la integración de la red de servicios de salud.

El plan de acción quedó constituido por 6 operaciones y 21 acciones de las cuales dos son acciones de demanda para CORPOSALUD Aragua y la alcaldía del municipio Santos Michelena (Tabla 5). Se procedió a seguir el análisis de la motivación de los actores. En la mayoría de las operaciones los actores tienen una motivación entre alta y media a favor.

La operación "diseñar el sistema de referencia y contra-referencia del municipio" se revela con baja viabilidad inicial, dada la forma como surgió la Misión Barrio Adentro en el país en paralelo a la red de servicios existentes. Sin embargo, se considera la posibilidad de resolver el impasse con una estrategia de negociación, que abra la discusión dirigida al entendimiento 9,24. 
Resumen de las operaciones para cada nudo crítico, plan de acción, Parroquia Capital, Municipio Santos Michelena, Estado de Aragua, Venezuela, año 2007.

Nudo crítico
NC1: insuficiente organización y participación
comunitaria en acciones de promoción de la salud

NC2: desintegración de las acciones en los diferentes niveles de atención de la red de salud (trabajo desarticulado)

NC3: fragmentación, desarticulación de la red de sistemas y servicios de salud

NC4: insuficiente número de recursos humanos cualificados en algunos de los niveles de atención para dar respuesta a la población de la que son responsables

NC5: baja capacidad tecnológica (insuficiente equipamiento e insumos en algunos de los establecimientos de salud de la parroquia)

\section{Operaciones}

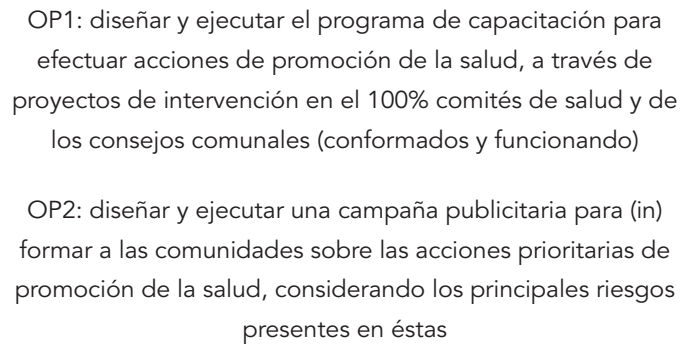

OP3: diseñar el sistema de referencia y contra-referencia del municipio

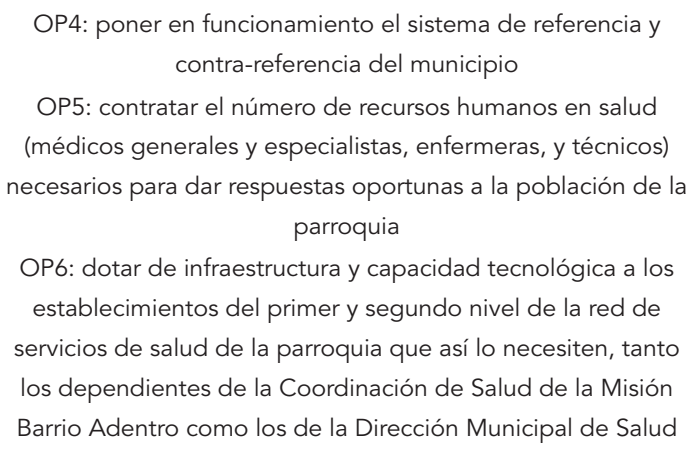

Fuente: plan de acción diseñado con los actores de la Parroquia Capital, Municipio Santos Michelena, Estado de Aragua, Venezuela, 2007.

Los actores prefirieron denominar la operación de conflicto como operación a ser negociada. Este punto conlleva la relevancia del enfoque comunicativo, que depende de la capacidad de los actores para asumir los procesos de negociación como procesos de aprendizaje. Esta es una de las fortalezas del equipo de salud de la parroquia, y confirma la importancia de que el plan sea una apuesta construida con la participación de la mayor parte de los actores, donde se dé lo que Rivera ${ }^{3}$ denomina una interacción lingüísticamente mediada.

\section{Conclusión}

El alcance de una mayor equidad en salud es uno de los principios que rigen la legislación venezolana.

Venezuela ha desarrollado diversas estrategias orientadas a la búsqueda de la operacionalización de la equidad en salud, lo que Matus 4,32 denomina reglas básicas o leyes, o sea, el marco legal vigente. El planeamiento en salud tiene un papel fundamental en la implementación de esas estrategias.

La adaptación del enfoque del PES en el nivel local en salud constituye una herramienta potencializadora en la inclusión de actores, sea en la identificación, sea en el enfrentamiento de problemas de salud, principalmente cuando el proceso es articulado con prioridades nacionales. El enfoque comunicativo, combinado con la perspectiva de equidad dificulta la cosificación de los actores y exige al mismo tiempo la incorporación de las diferentes opiniones y argumentos en el proceso de planeamiento, así como la incorporación de las poblaciones menos favorecidas.

Las herramientas utilizadas en la estratificación de las localidades permitieron articular la epidemiología y el planeamiento en salud, potencializando la explicación de la situación de salud.

La combinación del análisis de las necesidades relativas de salud y el análisis de la motivación del potencial participativo de los actores-clave 
conllevó una respuesta muy buena al problema sobre cómo priorizar las unidades objetivo, incorporando criterios de equidad en la aplicación de ciertas metodologías que, como ésta, están siendo probadas y que buscan contribuir con la mejoría de la salud de las poblaciones.

\section{Resumen}

El artículo discute los resultados de la operacionalización del Planeamiento Estratégico Situacional (PES) adaptado al nivel local en salud, considerando el enfoque comunicativo y la dimensión de la equidad en una parroquia en Venezuela. Se utilizaron dos criterios innovadores: la estimativa de las necesidades de salud y el análisis del potencial participativo de los actores. Los problemas identificados fueron relacionados con los artículos referentes a derechos de la Constitución venezolana. Se midieron inequidades con indicadores de salud, asociados al problema seleccionado; se incorporaron criterios de equidad en las propuestas de acción y elementos comunicativos. Se dio prioridad al problema "baja capacidad resolutiva de la red de servicios de salud" y se seleccionaron cinco nudos críticos para el plan de acción, que quedó constituido por 6 operaciones y 21 acciones. Se concluyó que la articulación de la epidemiología y planeamiento amplía la explicación situacional. La incorporación del enfoque comunicativo y de la dimensión de equidad al PES permite potencializar la gestión en salud y contribuir a la disminución de las brechas por inequidad.

Planificación en Salud; Inequidad Social; Condiciones Sociales; Participación Ciudadana
Se concluye que la incorporación del enfoque comunicativo y de la dimensión de la equidad al PES permite potencializar la gestión en salud y contribuir a la disminución de las brechas por inequidad.

\section{Colaboradores}

H. L. Heredia-Martínez realizó la concepción del proyecto, recogida, análisis e interpretación de los datos y redacción del artículo. E. Artmann contribuyó con la concepción del proyecto, análisis e interpretación de los datos y redacción del artículo. S. M. Porto participó en la concepción del proyecto, análisis e interpretación de los datos y revisión de la version en español. 


\section{Referencias}

1. Artmann E. O Planejamento Estratégico Situacional no nível local: um instrumento a favor da visão multisetorial. Rio de Janeiro: Instituto Alberto Luiz de Coimbra de Pós-graduação e Pesquisa de Engenharia, Universidade Federal do Rio de Janeiro; 2000. (Série Desenvolvimento Local; Cadernos da Oficina Social, 3).

2. Giovanella Lígia. As origens e as correntes atuais do enfoque estratégico em planejamento de saúde na América Latina. Cad Saúde Pública 1991; 7:26-44.

3. Rivera FJU. Agir comunicativo e planejamento social: uma crítica ao enfoque estratégico. Rio de Janeiro: Editora Fiocruz; 1995.

4. Matus C. Política, planejamento \& governo. 2a Ed. Brasília: Instituto de Pesquisa Econômica Aplicada; 1996.

5. Rivera FJU, Artmann E. Planejamento e gestão em saúde: flexibilidade metodológica e agir comunicativo. In: Rivera FJU, organizador. Análise estratégica em saúde e gestão pela escuta. Rio de Janeiro: Editora Fiocruz; 2003. p. 17-35.

6. Artmann E. O Planejamento Estratégico Situacional: a trilogia matusiana e uma proposta para o nível local de saúde (uma abordagem comunicativa) [Disertación de Maestría]. Rio de Janeiro: Escola Nacional de Saúde Pública, Fundação Oswaldo Cruz; 1993.

7. Artmann E. Interdisciplinaridade no enfoque intersubjetivo habermasiano: reflexões sobre planejamento e AIDS. Ciênc Saúde Coletiva 2001; 6 : 183-95.

8. Artmann E, Rivera F. Humanização no atendimento e gestão comunicativa. In: Deslandes SF, organizadora. Humanização dos cuidados em saúde: conceitos, dilemas e práticas. Rio de Janeiro: Editora Fiocruz; 2006. p. 205-31.

9. Habermas J. Teoría de la acción comunicativa. Madrid: Taurus; 1987.

10. Rawls J. Uma teoria da justiça. São Paulo: Editora Martins Fontes; 1997

11. Sem AK. Desigualdade reexaminada. Rio de Janeiro: Editora Record; 2001.

12. Starfield B. Improving equity in health: a research agenda. Int J Health Serv 2001; 13:545-66.

13. Porto S. Equidade na distribuição geográfica de recursos em saúde: uma contribuição para o caso brasileiro [Tese de Doutorado]. Rio de Janeiro: Escola Nacional de Saúde Pública, Fundação Oswaldo Cruz; 1997.

14. Porto SM. Equidad y distribución de recursos financieros en los sistemas de salud. Cad Saúde Pública 2002; 18:939-57.

15. Porto S, Martins M, Travassos C, Viacava F. Avaliação de uma metodologia de alocação de recursos financeiros do setor saúde para aplicação no Brasil. Cad Saúde Pública 2007; 23:1393-404.

16. Artmann E, Azevedo CS, Sá MC. Possibilidades de aplicação do enfoque estratégico de planejamento no nível local de saúde: análise comparada de duas experiências. Cad Saúde Pública 1997; 13:723-40.

17. Ministerio de Salud y Desarrollo Social. Identificación y representación de las necesidades sociales. Modulo II. Caracas: Ministerio de Salud y Desarrollo Social; 2002.
18. Bergonzoli G. Sala situacional. Instrumento para la vigilancia de salud pública. Maracay: Editora IAESP; 2006.

19. Organización Panamericana de la Salud. Resúmenes metodológicos en epidemiología: análisis de la situación de salud. Bol Epidemiol (Wash) 1999; 20:1-3.

20. Castellanos PL. Perfiles de salud y condiciones de vida: una propuesta operativa para el estudio de inequidades de salud en América Latina. In: Anales del I Congreso Iberoamericano de Epidemiología. Granada: s.l.; 1992.

21. Castellanos PL. Epidemiología, salud pública, situación de salud y condiciones de vida. Consideraciones conceptuales. São Paulo: Faculdade de Ciências Médicas da Santa Casa de São Paulo; 1995.

22. Castellanos PL. Los modelos explicativos del proceso salud. Enfermedad: los determinantes sociales. Madrid: McGraw-Hill; 1998.

23. Habermas J. Pensamento pós-metafísico: estudos filosóficos. Rio de Janeiro: Tempo Brasileiro; 1990.

24. Habermas J. A inclusão do outro: estudos de teoria política. 2a Ed. São Paulo: Edições Loyola; 2004.

25. Viana SM, Nunes A, Silva-Santos JR, Barata R. Medindo as desigualdades em saúde no Brasil: uma proposta de monitoramento. Brasília: Organização Pan-Americana da Saúde/Instituto de Pesquisa Econômica Aplicada; 2001.

26. Junqueira V, Pessoto UC, Kayano J, Nascimento PR, Castro IEN, Rocha JL, et al. Equidad en la salud: evaluación de políticas públicas en Belo Horizonte, Minas Gerais, Brasil, 1993-1997. Cad Saúde Pública 2002; 18:1087-101.

27. Kozu KT, Godinho LT, Muniz MVF, Chiarioni P. Mortalidade infantil: causas e fatores de risco. Um estudo bibliográfico. http://www.medstudents. com.br/original/original/mortinf/mortinf.htm (accedido el 10/Mar/2010).

28. Carvalho A. Conselhos de saúde no Brasil [Dissertação de Mestrado]. Rio de Janeiro: Escola Nacional de Saúde Pública, Fundação Oswaldo Cruz; 1994.

29. Berrenchea J, Trujillo E, Chorny A. Implicaciones para la planificación y administración de los sistemas de salud. Medellín: Universidad de Antioquía; 1989.

30. Matus C. Sobre la teoría de las macroorganizaciones. Revista PES 1994; 3:3-5.

31. Matus C. La ciencia y la política (conferencia). Salud Colect 2007; 3:81-91.

32. Huertas F. El método PES. Entrevista con Matus. Caracas: Fondo Editorial Altadir; 1996.

33. Artmann E, Rivera FJU. A démarche stratégique (gestão estratégica hospitalar): um instrumento de coordenação da prática hospitalar baseado nos custos de oportunidade e na solidariedade. Ciênc Saúde Coletiva 2003; 8:479-99.

Recibido el 08/Nov/2009

Versión final presentada el 22/Mar/2010

Aprobado el 05/Abr/2010 